\section{Effects of presentation rate and prior knowledge of target identity in the repeated-presentations paradigm*}

\author{
THOMAS R. HERZOG \\ Grand Valley State College, College Landing, Allendale, Mich. 49401
}

Single letters were presented for varying numbers of repeated brief exposures. After each trial, the $\mathbf{S}$ identified only those letters perceived with certainty on the last presentation. Three interpresentation intervals were used: $0.5,2.0$, and 4.0 sec. The percentage of correct identifications (hits) decreased as interpresentation interval increased. Prior knowledge of the target facilitated performance in all conditions, with the greatest improvement for the 2.0 -sec interpresentation interval. The repetition curve relating hit rate to trial length showed a growth trend only in the absence of prior knowledge of the target. There was some suggestion of a decline in performance for longer trial lengths with the 0.5 -sec interpresentation interval. Possible effects on the repetition curve of attentional factors and the buildup of satiation were discussed.

Haber has demonstrated an effect of repeated brief presentations of constant duration on the percentage of correct reports of target letters or words (see Haber, 1969, for a review). As the number of repeated presentations of a given target is increased, the percentage of correct reports of the target also increases to an asymptote that depends on exposure duration. Herzog (1971) has shown that prior knowledge of the identity of single-letter targets may produce both a general facilitation in performance and an alteration in the shape of the repetition curve. The prior-knowledge curve is flat, with no growth trend. The interval between repeated presentations has never been precisely controlled in the past and has varied widely among studies. The experiment reported here examines both the shape of the repetition curve and the nature of the prior-knowledge effect when the presentation rate for repeated single-letter targets is precisely controlled.

\section{SUBJECTS}

Nine summer school students at the University of Rochester, seven females and two males, served as paid Ss. Each participated in eight 1 -h sessions.

$$
\text { APPARATUS }
$$

The stimuli were 12 capital letters (A, C, D, F, K, L, N, S, T, U, Y, and Z). They were constructed from "Letraset" dry-transfer letters (Futura Bold, 20-pt) and subtended $15 \mathrm{~min}$ of visual angle vertically at a $127-\mathrm{cm}$ viewing distance. The letters were presented one at a time in two

*This research was supported by a National Science Foundation postdoctoral fellowship to the author. The help and advice of Drs. Ralph Norman Haber and A. $O$. Dick of the University of Rochester is gratefully ack nowledged. channels of a three-field tachistoscope (Scientific Prototype Model GB). One of the stimulus channels was used to present the same letter on all but the last flash of a trial; the other channel was used to present either that same letter or a different letter on the last flash of the trial. The third channel, containing a centrally located fixation square $1.59 \mathrm{~cm}(0.625$ in.) on a side, was lighted whenever a letter was not being presented. Timing and sequencing of events within each trial was controlled by a PDP-8 computer, on-line with the tachistoscope. Stimulus and background fields were adjusted to appear equally bright to the $S$ during a trial. Luminances averaged 51.5 and $48.1 \mathrm{~mL}$ for the two stimulus fields and $36.5 \mathrm{~mL}$ for the background field. Viewing was binocular.

\section{PROCEDURE}

In each session, a modified staircase procedure was used to determine an exposure duration at which the $S$ could identify correctly a target presented once approximately $30 \%$ of the time. During these preliminary trials, the $S$ used the same set of for the experimental trials. The exposure duration determined from the preliminary trials was used for all presentations during the experimental trials. Exposure durations varied from 2.5 to $10.0 \mathrm{msec}$.

Each experimental trial consisted of from one to five presentations. The $\mathrm{S}$ was instructed to pay equal attention to all presentations of a trial but to report afterward on what he had seen on the last presentation only. He was informed in advance how many presentations each trial would contain so that he would know which presentation was to be the last. On half of the trials of each length, the response indicators as that given below same letter was shown on all presentations (regular trials); on the other half, a catch letter (a random selection of one of the 11 letters other than the scheduled target letter for that trial) was shown on the last presentation only (catch trials). The $S$ was informed of the possibility of catch letters and of their a priori probability of occurrence in the initial instructions.

After each trial, the $\mathbf{S}$ described what he had seen on the last presentation, using the following set of responses: blank-the $S$ saw nothing but a blank field; blur-the $S$ saw a stimulus, but it was not clear enough for him to identify as either a specific symbol or part of a specific symbol; part symbol, vague-the $S$ saw part of a specific symbol, but the part seen was not clear or sharply defined; part symbol, clear-the $S$ saw part of a specific symbol, and the part seen was clear or sharply defined; whole symbol, vague-the $S$ saw all of a specific symbol, but the symbol was not clear or sharply defined; whole symbol, clear-the $S$ saw all of a specific symbol, and the symbol was clear or sharply defined. Whenever the $S$ made a part- or whole-symbol response, he was required to identify the specific letter he had seen. A conservative criterion for reporting was encouraged by specifically instructing the $S$ to use one of the identification responses only when he was certain which letter had been shown on the last presentation of the trial. The $\mathrm{S}$ was given full feedback after responding, including the identity of the target and of the catch letter, if one was shown. The $S$ initiated each trial by pressing a button; the entire sequence of presentations then occurred automatically under the control of the computer.

The independent variables were presentation rate (interpresentation intervals of $0.5,2.0$, and $4.0 \mathrm{sec}$ ), prior target uncertainty (no advance information and prior knowledge of the scheduled target letter), and trial length (1-5 presentations). Prior knowledge was provided by telling the $S$ before the trial what the scheduled target letter was. He was never told in advance that a catch letter would be shown. Each session contained 120 experimental trials. Within each session, presentation rate was held constant within blocks of 40 trials each. Within each such block, one level of prior target uncertainty was maintained within subblocks of 20 trials each. Order of occurrence of the three presentation rates within a session was balanced across sessions for each $S$. The no-information subblock of trials occurred first for 


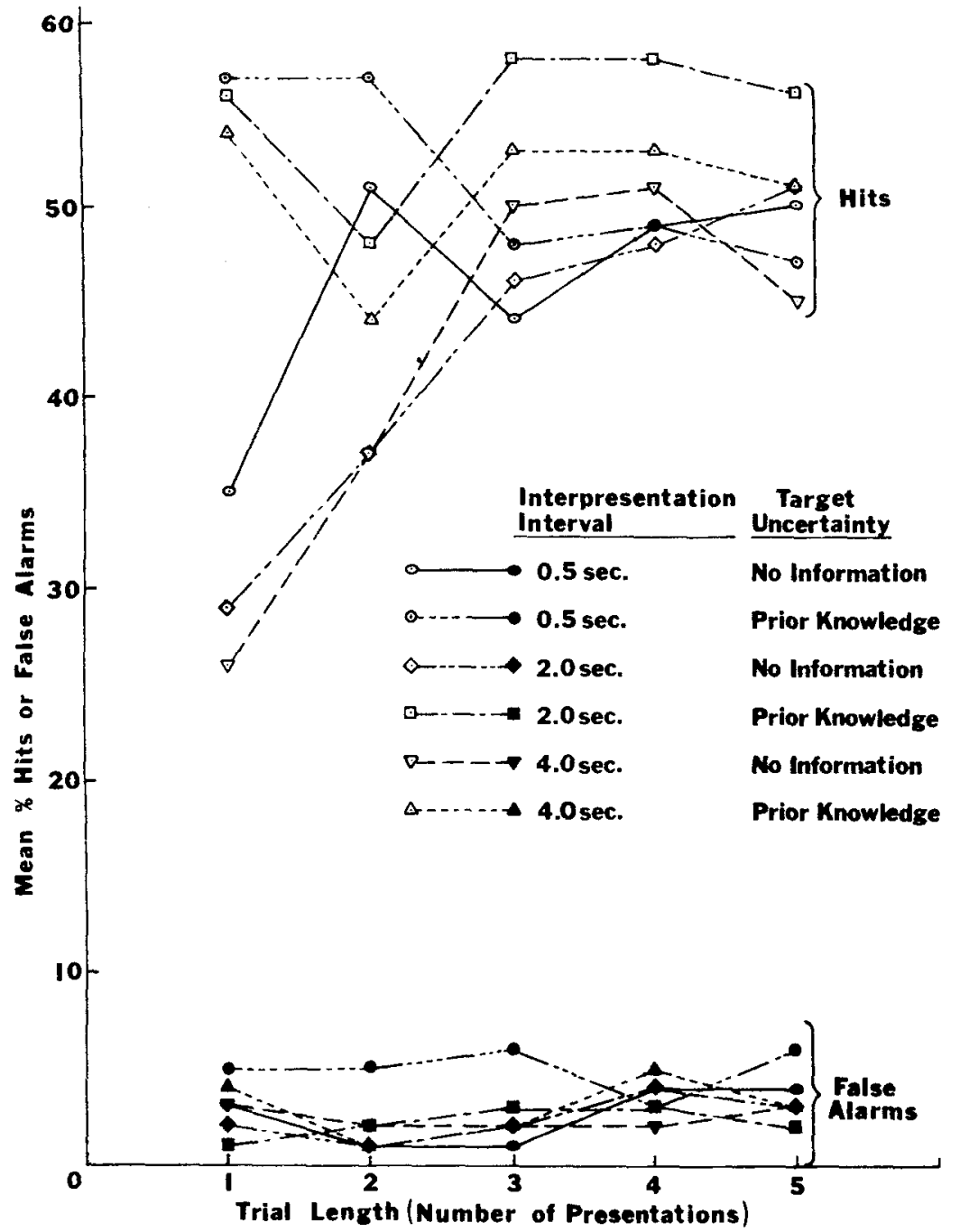

Fig. 1. Mean percentage of hits (top six functions) and false alarms (bottom six functions) as a function of presentation rate (interpresentation interval), target uncertainty, and trial length (number of presentations). (Each point is the mean percentage for the nine Ss.)

each presentation rate in odd-numbered experimental sessions; the prior-knowledge subblock occurred first in even-numbered sessions. Within each subblock, trial length was randomized, with the restrictions that no more than three consecutive trials of a given length were allowed and that each trial length occur four times. Of those four trials, two were catch trials. A further restriction on the randomness of trial order within subblocks was that a letter could not be the target on a given trial if it had appeared as either a target or a catch letter on the preceding trial.

The first two sessions for each $\mathrm{S}$ were practice sessions (data not analyzed). Across the last six sessions, each of the 12 stimulus letters was used once as the target letter for each combination of presentation rate,

of 270 possible cases, the percentage of false alarms was zero), it was not possible to estimate reliably the signal-detection sensitivity measure, $d^{\prime}$. Therefore, the results are not reported in terms of $\mathrm{d}^{\prime}$ scores.

\section{RESULTS}

Figure 1 presents the results for hits and false alarms. A repeated-measures analysis of variance of the percent false-alarm scores of individual Ss yields a significant effect of presentation rate, $F(2,16)=3.94$, $\mathrm{p}=.04$. The mean false-alarm percentages for the three presentation rates are 3.6, 2.1, and 2.5 for interpresentation intervals of $0.5,2.0$, and $4.0 \mathrm{sec}$, respectively. No other main effects or interactions approach significance.

A repeated-measures analysis of variance of the percent hit scores of individual $\mathrm{Ss}$ yields a significant effect of prior target uncertainty, $F(1,8)=$ $45.92, \mathrm{p}=.0003, \mathrm{a}$ significant Presentation Rate by Prior Target Uncertainty interaction, $F(2,16)=$ $4.04, p=.04$, and a significant Prior Target Uncertainty by Trial Length interaction, $\mathrm{F}(4,32)=6.27, \mathrm{p}=.001$. The mean percentages of hits for the two prior-target-uncertainty conditions are 43.3 and 52.6 for no information and prior knowledge, respectively. Table 1 , which contains the mean percentages of hits for all combinations of presentation rate and prior target uncertainty, shows the nature of the Presentation Rate by Prior Target Uncertainty interaction. In the no-information condition, there is a decline in hit rate as presentation rate becomes slower. However, in the prior-knowledge condition, there is first an increase and then a decrease in hit rate as presentation rate becomes slower. The net effect is that prior knowledge produces the greatest facilitation in hit rate with the 2.0 -sec interpresentation interval (an increase of $13.2 \%$ ) and lesser gains with the 0.5 - and $4.0-\mathrm{sec}$ intervals (increases of $5.7 \%$ and $9.2 \%$, respectively). The nature of the Prior Target Uncertainty by Trial Length interaction is clear from Fig. 1. As a group, the three no-information curves tend to grow as a function of trial length, reaching asymptote at a trial length of four presentations. On the other hand, the prior-knowledge curves remain relatively stable across trial lengths. These impressions are confirmed by trend analyses of the individual curves. Among the no-information curves, those for interpresentation intervals of 2.0 and $4.0 \mathrm{sec}$ both contain increasing

Table 1

Mean Percentages of Hits for Each of Three Presentation Rates (Interpresentation Intervals) and Each of Two Prior Target Uncertainty Conditions

\begin{tabular}{cccc}
\multicolumn{4}{c}{ Uncertainty Conditions } \\
\hline $\begin{array}{c}\text { Prior } \\
\begin{array}{c}\text { Target } \\
\text { Uncertainty }\end{array}\end{array}$ & $\begin{array}{l}\text { Interpresentation Interval } \\
\text { Sec }\end{array}$ & $\begin{array}{c}2.0 \\
\text { Sec }\end{array}$ & $\begin{array}{c}4.0 \\
\text { Sec }\end{array}$ \\
\hline $\begin{array}{c}\text { No } \\
\text { Information } \\
\begin{array}{c}\text { Prior } \\
\text { Knowledge }\end{array}\end{array}$ & 46.0 & 42.2 & 41.6 \\
\hline
\end{tabular}


linear trends $(\mathrm{p}=.008$ and .02 , respectively); the latter also contains a quadratic component $(p=.004)$. There are no significant trends among the prior-knowledge curves. Of interest are two trends that approach significance, both for the fastest presentation rate: a cubic trend for the no-information curve $(\mathrm{p}=.08)$ and a decreasing linear trend for the prior-knowledge curve $(p=.06)$. DISCUSSION

Since the false-alarm rates in this experiment are extremely low, and since the one significant result involving false alarms (the main effect of presentation rate) is not paralleled in the hit data, it is probable that variation in false-alarm rates is not critical in interpreting these results. The hit rates of this experiment may be taken as reasonably valid indicators of the effects of the independent variables when false-alarm rates are held constant. 1

The main effect of prior target uncertainty on hit rate and the interaction of target uncertainty and trial length both replicate the earlier findings of Herzog (1971). That is, prior knowledge increases perceptual sensitivity to an asymptotic level that is maintained across the trial lengths investigated. Without prior knowledge, perceptual sensitivity is very low on the initial presentation and grows with repeated presentations of the target. The growth in target hit rate as a function of repeated presentations in the no-information condition, in the absence of any change in false-alarm rate, supports Haber's interpretation of the repetition effect as being perceptual in nature. Herzog's (1971) findings had raised some doubt on this point. He obtained results similar to those presented here in a pilot study, but in his main experiment the growth effect in the no-information condition was shown to have been caused by a progressive liberalization of the S's criterion for reporting as a function of repeated presentations. Perhaps the most prudent interpretation of all findings to date is that sometimes the growth effect represents a change in perceptual sensitivity, sometimes a change in criterion for reporting, and the conditions favoring one effect or the other remain to be discovered. In any event, the evidence for an effect of prior knowledge on perceptual sensitivity is strengthened by the findings of this experiment.

The interaction of presentation rate and prior target uncertainty can be explained in practical terms. In the no-information condition, repeated presentations of a target result in the buildup of some internal priming or facilitatory process. The faster the presentation rate, the less time is available for this priming to dissipate between presentations. Hence, an inverse monotonic relationship between interpresentation interval and hit rate is to be expected and is, in fact, obtained. To be maximally effective, prior knowledge of the target must be accompanied by active rehearsal of the advance information on the part of the $S$. The introspective reports of Ss indicate that they have little or no time for rehearsal when the interpresentation interval is $0.5 \mathrm{sec}$, and they become bored and lose interest in rehearsal when the interpresentation interval is $4.0 \mathrm{sec}$. A $2.0-\mathrm{sec}$ interpresentation interval strikes an optimum balance between opportunity and motivation to rehearse. Hence, as Table 1 indicates, prior knowledge yields the greatest facilitation in performance with a 2.0 -sec interpresentation interval.

Perhaps the most intriguing finding of this experiment is the suggestion in the data that something unusual is occurring in the repetition curves for the $0.5 \mathrm{sec}$ interpresentation interval. As the trend analyses indicate, the 0.5 -sec curves tend to be different in shape from the 2.0- and 4.0-sec curves, which are quite similar. The 0.5-sec curves are the only ones that show a (nearly) significant decline in hit rate. The no-information curve grows to a peak at the second presentation, declines on the third presentation, and recovers on the last two presentations (a cubic trend). The prior-knowledge curve is at its peak on the first presentation and declines thereafter (a decreasing linear trend). Kaplan (Pomerantz, Kaplan, \& Kaplan, 1969) has postulated that not only does facilitation build up in an internal representation of the target as a function of repeated presentations, but satiation also accumulates at a slower rate. Satiation effects manifest themselves in a decline in the repetition curve. Such a decline should occur earlier with faster presentation rates and/or prior knowledge of the target. In the present experiment, the fastest presentation rate yields a decline in performance after the second repetition in the no-information condition and after the initial presentation in the prior-knowledge condition. Perhaps, therefore, the 0.5 -sec curves are being influenced by satiation processes that do not build up sufficiently to affect the slower presentation rates. If so, it is clear that this experiment did not sample in an appropriate range of presentation rates to provide an adequate test of Kaplan's model. Future experiments of this type might profit from an examination of interpresentation intervals between 0.5 and 2.0 sec.

\section{REFERENCES}

HABER, R. N. Repetition, visual persistence, visual noise, and information processing. In $\mathrm{K}$. N Leibovic (Ed.) Information processing in the nervous system Columbus: Springer, 1969. Pp. $121-140$.

HER2OG, T. R. Effects of amount and kind of prior target-uncertainty reduction in the repeated presentations paradigm. Perception \& Psychophysies, 1971, 10 , 273-277.

POMERANTZ, J. R., KAPLAN, S., \& KAPLAN, R. Satiation effects in the perception of single letters. Perception \& Psychophysies, 1969, 6, 129-132. NOTE

1. If the number of hits and false alarms for each $S$ in each experimental condition is increased by one and $d^{\prime}$ scores are then computed from the resulting hit rates and false-alarm rates, the major conclusions of the experiment are not changed. 